\title{
¿Cómo perciben los estudiantes la adquisición de competencias? Análisis comparado: Teorías de la Comunicación en la Universidad Pompeu Fabra
}

\author{
Lorena GómEZ-PUERTAS \\ Universidad Pompeu Fabra \\ lorena.gomez@upf.edu \\ Carles RocA-Cuberes \\ Universidad Pompeu Fabra \\ carles.roca@upf.edu \\ Frederic GUERRERO-SOLÉ \\ Universidad Pompeu Fabra \\ frederic.guerrero@upf.edu
}

\begin{abstract}
Resumen:
El aprendizaje por competencias es el núcleo del paradigma docente del EEES. Este artículo recoge los resultados del estudio comparado de la percepción de los estudiantes de Teorías de la Comunicación sobre la adquisición de competencias en los grados de Comunicación Audiovisual, Publicidad y Periodismo de la Universidad Pompeu Fabra. La investigación recurre a dos técnicas, la encuesta y el grupo de discusión, que permiten analizar la dificultad de comprensión de determinadas competencias y la percepción de los estudiantes sobre su adquisición, utilidad, definición y posible evaluación.
\end{abstract}

Palabras clave: adquisición de competencias; aprendizaje por competencias; métodos de investigación en comunicación; teorías de la comunicación, análisis comparativo

How students perceive their competence development? Comparative analysis: Communication Theories at Pompeu Fabra University

\begin{abstract}
:
The core of the educational paradigm of ESHE is competence learning. This paper contains the results of the comparative analysis of the self-perception of competence development by Communication Theories students in the degree courses AudioVisual Communication, Advertising and Public Relations, and Journalism at the Pompeu Fabra University. Two complementary techniques were employed for the study, survey and focus group, in order to facilitate an analysis of comprenhension difficulties for some competences and student perception of their development, utility, definition and possible evaluation.
\end{abstract}

Key Words: competence development; competence learning; communication research methods; communication theory; comparative analysis

\section{Referencia normalizada:}

Gómez-Puertas, L., Roca-Cuberes, C. y Guerrero-Solé, F. (2014): ¿Cómo perciben los estudiantes la adquisición de competencias? Análisis comparado: Teorías de la comunicación en la universidad Pompeu Fabra. Historia y Comunicación Social. Vol. 19. Núm. Especial Febrero. Págs. 313-326.

Sumario: 1.Introducción 2.Metodología 3.Resultados de la investigación 3.1.Encuesta. 3.2.Grupos de discusión 3.2.1.Competencias transversales 3.2.2.Competencias específicas 4. Conclusiones y discusión 5.Bibliografía 


\section{Introducción}

La universidad española se halla inmersa en el proceso de transición al EEES (Ministerio de Educación Cultura y Deporte, 2003) que le ha llevado a adoptar un modelo de formación académica donde se sitúa al estudiante y su aprendizaje en el centro de la institución.

Este proceso requiere un cambio tanto de mentalidad como de procedimientos por parte de los actores involucrados en su implementación (De la Cruz, 2003). En primer lugar porque recae sobre el aprendizaje por competencias la base del nuevo modelo de formación académica, debiéndose integrar tanto las competencias transversales propias de la educación general, como aquellas específicas relacionadas con ámbitos profesionales delimitados (Pedró, 2005; Martínez-Cocó et al., 2008; Rauhvargers, 2011).

En segundo lugar, porque supone profundos cambios en las metodologías de enseñanza y evaluación. Así, se fomenta una enseñanza focalizada en el aprendizaje autónomo del estudiante, que exige nuevos métodos y estrategias de trabajo (solución de problemas, aprendizaje cooperativo o por proyectos, estudio de casos, etc.) y al mismo tiempo, deben diseñarse sistemas de evaluación, con modalidades alternativas (formativa, coevaluación, autoevaluación, etc.), que permitan valorar la adquisición de competencias como expresión del aprendizaje del estudiante (Biggs, 2003; Angulo et al.,2006). Es, por tanto, necesario pasar de la evaluación del aprendizaje a la evaluación para el aprendizaje y a la evaluación como aprendizaje (Gibbs y Simpson, 2004; Stiggins, 2006), aún asumiendo las dificultades que se reconocen en la factibilidad de evaluar las competencias (Zabala y Arnau, 2008; Álvarez, 2008).

La Universitat Pompeu Fabra ha implementado los nuevos Grados de Comunicación Audiovisual y Publicidad y Relaciones Públicas, desde el curso 2008-2009, y un año más tarde, el de Periodismo. En este estudio se analiza si la transición a dichos grados ha generado un aprendizaje de calidad en los estudiantes, atendiendo el reconocimiento de su nivel de adquisición de competencias en una materia común, Teorías de la Comunicación.

Es cada vez más notable la toma en consideración de la percepción de los estudiantes sobre todos los aspectos de su experiencia en la educación superior, convertidos de esta manera en indicadores de la calidad de la universidad (Arnold et al.,1999; Hill et al.,2003). En esta línea, el procedimiento metodológico habitualmente usado ha sido el de la encuesta. En particular, se ha utilizado en algunos estudios el Student Engagement Questionnaire para evaluar diferentes estudios de grado (Kember y Leung, 2005a; 2005b; Leung y Kember, 2005; 2006), donde los resultados mostraron, por un lado, que la calidad del entorno de enseñanza y aprendizaje afecta de manera significativa al desarrollo de las competencias generales, y por otro lado, durante un periodo de cinco años, sirvieron para llevar a cabo iniciativas de mejora de la calidad de la enseñanza. 
Sin embargo, uno de los principales problemas al que nos enfrentamos a la hora de analizar la adquisición de competencias por parte de los estudiantes es la disparidad conceptual, ambigüedad y amplitud semántica asociadas a la misma noción de competencia (Boni y Lozano, 2006:829; Alonso et al.,2009:65-68). A efectos de esta investigación, y de acuerdo al modelo implementado en la Universidad Pompeu Fabra, se suscribe la definición de competencia propuesta por la Agència de Qualitat Universitària de Catalunya. Así, una competencia es una "combinación de saberes técnicos, metodológicos y participativos que se actualizan en una situación y un momento particulares", fórmula que, como podemos ver, no incluye aspectos teóricos, y que "implican el uso integrado de conocimientos, habilidades y actitudes en la acción. Por su naturaleza, sólo se podrán alcanzar en los estadios finales del proceso educativo" (AQU,2004:16). En lo referente a la clasificación de competencias a adquirir en las materias de Teorías de la comunicación, se ha adoptado la ordenación básica entre competencias transversales, y competencias específicas (Figura 1).

\section{Figura 1. Competencias de la asignatura Teorías de la Comunicación}

\section{COMPETENCIAS TRANSVERSALES}

1. Obtener el conocimiento general básico en comunicación.

2. Adquirir la capacidad de análisis y síntesis de la información.

3. Ser capaz de trabajar en equipo.

4. Saber ser crítico con el trabajo y los resultados de su entorno.

5. Potenciar la capacidad crítica necesaria para reflexionar sobre lo que se hace, para generar nuevas acciones y dar sentido a las propias acciones.

6. Actuar con libertad y corresponsabilidad, asumiendo referentes éticos, valores y principios consistentes.

7. Desarrollar las capacidades empáticas y potenciar la inteligencia emocional para la valoración de situaciones de los objetos de su entorno.

8. Ser capaz de aprender a aprender.

COMPETENCIAS ESPECÍFICAS:

9. Obtener el conocimiento y la visión crítica metateórica de las diferentes corrientes de las teorías de la comunicación y de sus fundamentos metodológicos.

10. Adquirir la capacidad de análisis crítico y comprensivo de los fenómenos comunicativos.

11. Saber buscar las claves interpretativas de cualquier fenómeno social, económico, productivo, etc.

12. Comprender que cualquier fenómeno humano es generado en el tiempo por agentes, por relaciones sociales, por necesidades, por intereses, por acciones racionales, etc. y que es susceptible de tener una naturaleza reactiva hacia los parámetros presentes en el propio contexto en que se localiza.

13. Desarrollar la imaginación y el pensamiento productivo con flexibilidad y fluidez para madurar actitudes de apertura a nuevas informaciones, situaciones y metodologías, y plantear problemas de investigación.

14. Conocer los valores sociales en cada momento para adecuar los mensajes a estos valores.

15. Ser capaz de aplicar los conocimientos teóricometodológicos

16. Adquirir la capacidad de plantear un diseño de investigación en el campo de la comunicación.

17. Saber aplicar la metodología de investigación científica para el análisis de diferentes fenómenos comunicativos.

18. Comprender el proceso de investigación en sus diferentes fases y cómo están estas interrelacionadas.

19. Ser capaz de evaluar propuestas de investigación. 
Estas competencias son comunes a las materias de Teorías de la comunicación, salvo en su formulación exclusivamente teórica en el Grado en Periodismo, donde tan sólo se incluyen las 15 primeras, descartando aquellos saberes que derivan del aprendizaje de carácter técnico y metodológico y conforman las cuatro últimas competencias específicas.

\section{Metodología}

Con el objetivo de preguntar a los estudiantes en qué grado creían haber adquirido cada una de las competencias propuestas por la asignatura cursada, se han utilizado en paralelo dos técnicas de investigación: la encuesta y el grupo de discusión (focus group).

En la encuesta los estudiantes deben determinar su percepción respecto al grado de adquisición de cada una de las competencias propuestas, de acuerdo a la siguiente escala de Likert: 5 (totalmente), 4 (bastante), 3 (suficiente), 2 (poco) y 1 (nada). Mientras que la técnica del grupo de discusión permite indagar cuáles son los argumentos proporcionados por los estudiantes sobre su adquisición de competencias: no sólo en qué medida, sino también de qué modo o bajo que motivación. La respuesta abierta e interactiva entre los componentes del grupo puede poner al descubierto conflictos y desacuerdos subyacentes, desconocidos por el investigador o inclusive por los propios miembros del grupo (Parahoo, 1999).

Para este estudio, las encuestas de autopercepción de los estudiantes sobre las competencias adquiridas se llevaron a cabo en dos fases, de acuerdo al calendario de implantación de los tres grados: en mayo de 2010, para los grados de Comunicación Audiovisual y Publicidad y Relaciones Públicas, y en diciembre de 2010, para el grado de Periodismo, coincidiendo con la finalización del periodo lectivo (Figura 2). La consistencia interna de las respuestas viene proporcionada por el Alfa de Cronbach, que varía entre el 0,81 (Comunicación Audiovisual) y el 0,88 (Publicidad y Relaciones Públicas).

Figura 2. Participantes en las encuestas de autopercepción de las competencias

\begin{tabular}{c|c|c|c|} 
& $\begin{array}{c}\text { Alumnos } \\
\text { totales }\end{array}$ & Participantes encuesta & Porcentaje \\
\hline Comunicación Audiovisual & 71 & 46 & $64,8 \%$ \\
\hline Publicidad y RRPP & 80 & 60 & $75 \%$ \\
\hline Periodismo & 71 & 67 & $94,4 \%$ \\
\hline
\end{tabular}

Por su parte, cada uno de los grupos de discusión contó con 8 participantes, que denominaremos con la siguiente nomenclatura: Sujeto X(1-8) - (CAU/PyRP/PER) y du duración osciló entre los 60 y 90 minutos, debidamente grabados y transcritos. 


\section{Resultados de la investigación}

\subsection{Encuestas}

Según las encuestas realizadas, los estudiantes de los tres grados perciben un alto grado de adquisición de las competencias propuestas por la materia, con una media que supera los 3 puntos sobre 5 (Figura 3 ).

Figura 3. Nivel medio de percepción de la adquisición de las competencias

\begin{tabular}{c|c|c|c} 
& $\begin{array}{c}\text { Competencias } \\
\text { transversales }\end{array}$ & $\begin{array}{c}\text { Competencias } \\
\text { específicas }\end{array}$ & Media global \\
\hline Comunicación Audiovisual & 3,62 & 3,73 & 3,68 \\
\hline Publicidad y RRPP & 3,45 & 3,32 & 3,37 \\
\hline Periodismo & 3,18 & 3,24 & 3,21 \\
\hline
\end{tabular}

Al revisar la polarización de las respuestas, el grado de Periodismo es el que concentra más respuestas de no haber adquirido en absoluto alguna competencia (4\%), seguido de Publicidad y Relaciones Públicas (2\%) y al nulo registro en Comunicación Audiovisual, cuyo dato más bajo contempla un 1,2\% de respuestas con valor 1 (Figura 4). Estos datos corroboran la percepción de la adquisición de competencias más positiva por parte de los estudiantes de Comunicación Audiovisual (con un $94,4 \%$ de las respuestas entre los niveles 3 y 5 , tan sólo el 5,6\% entre el 1 y 2), en contraste con la más crítica adoptada por los discentes en Periodismo (con un 22,7\% de respuestas negativas).

Figura 4. Distribución de las respuestas por grados

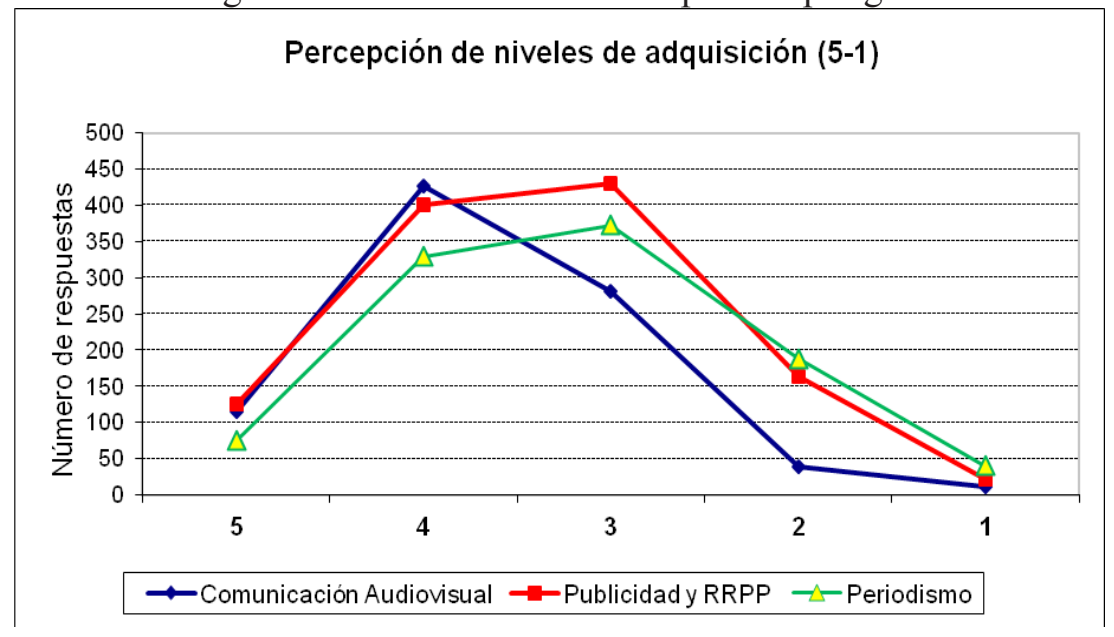

En efecto, se observa una tendencia paralela entre Comunicación Audiovisual y Publicidad y Relaciones Públicas, en las competencias transversales que más perciben haber adquirido (3 y 4) (Figura 5). Y pese a coincidir los tres grados en la percepción 
de las competencias transversales que menos han adquirido, esto es, aquéllas relacionadas con cuestiones éticas, emocionales y de aprendizaje $(6,7,8)$, se establecen ciertos puntos de divergencia entre los estudiantes de Periodismo y sus homólogos. Además de desmarcarse de la percepción de elevada adquisición de las competencias transversales (destacan las 5 y 4), los estudiantes de Periodismo marcan oscilaciones diversas en cuanto a la adquisición de competencias específicas.

Figura 5. Media de adquisición de competencias (1-5)

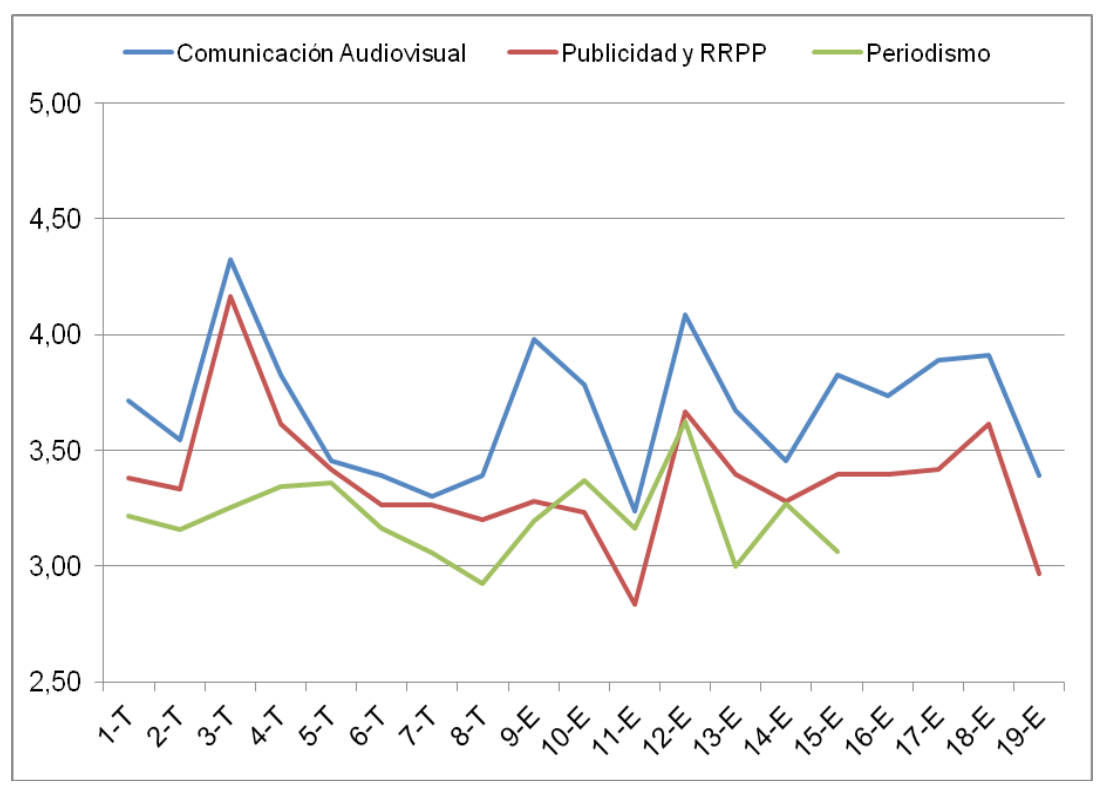

\begin{tabular}{|c|c|c|c|}
\hline \multicolumn{2}{|c|}{$\begin{array}{c}\text { Adquisición } \\
\text { percibida }\end{array}$} & Máxima & Mínima \\
\hline $1(\mathrm{~T})$ & 3,72 & 3,38 & 3,22 \\
\hline $2(\mathrm{~T})$ & 3,54 & 3,33 & 3,16 \\
\hline $3(\mathrm{~T})$ & 4,33 & 4,17 & 3,25 \\
\hline $4(T)$ & 3,83 & 3,62 & 3,34 \\
\hline $5(\mathrm{~T})$ & 3,46 & 3,42 & 3,36 \\
\hline $6(\mathrm{~T})$ & 3,39 & 3,27 & 3,16 \\
\hline $7(\mathrm{~T})$ & 3,30 & 3,27 & 3,06 \\
\hline $8(\mathrm{~T})$ & 3,39 & 3,20 & 2,92 \\
\hline
\end{tabular}

\begin{tabular}{c|c|c|c} 
& & & \\
& & & \\
\hline $9(\mathrm{E})$ & 3,98 & 3,28 & 3,19 \\
\hline $10(\mathrm{E})$ & 3,78 & 3,23 & 3,37 \\
\hline $11(\mathrm{E})$ & $\mathbf{3 , 2 4}$ & $\mathbf{2 , 8 3}$ & 3,16 \\
\hline $12(\mathrm{E})$ & 4,09 & 3,67 & 3,63 \\
\hline $13(\mathrm{E})$ & 3,67 & 3,40 & $\mathbf{3 , 0 0}$ \\
\hline $14(\mathrm{E})$ & 3,46 & 3,28 & 3,27 \\
\hline $15(\mathrm{E})$ & 3,83 & 3,40 & 3,06 \\
\hline $16(\mathrm{E})$ & 3,74 & 3,40 & \\
\hline $17(\mathrm{E})$ & 3,89 & 3,42 & \\
\hline $18(\mathrm{E})$ & 3,91 & 3,62 & \\
\hline $19(\mathrm{E})$ & 3,39 & 2,97 & \\
\hline
\end{tabular}


Al margen del consenso unánime en la percepción de la competencia específica 12 como la más adquirida, destaca el contraste establecido para la competencia relativa al contenido teórico (9) entre los estudiantes de Comunicación Audiovisual, que la perciben como una de las más adquiridas, y los de Publicidad, que la sitúan entre las que menos. Una divergencia que se reproduce para las competencias de análisis crítico y pensamiento flexible (10 y 13). Si coinciden, en cambio, en la percepción de las competencias específicas menos adquiridas, las dedicadas a la comprensión de los fenómenos comunicativos (11 y 19), destacadas en sentido contrario por los estudiantes de Periodismo.

Por último, cabe señalar la desviación interna respecto a la media de adquisición percibida de las competencias transversales y específicas de cada grado (Figura 6). Así, se observa una desviación similar entre los grados de Comunicación Audiovisual y Publicidad, con un significativo diferencial entre las competencias transversales (esp. valoración positiva del Trabajo en equipo) y una desigual percepción de las competencias específicas adquiridas (esp. contrasta la alta valoración de competencias de tipo comprensivo, 12 y 18, frente a la menor adquisición percibida de competencias analíticas, 11, 14 y 19).

Figura 6. Desviaciones de la media en la percepción de competencias adquiridas
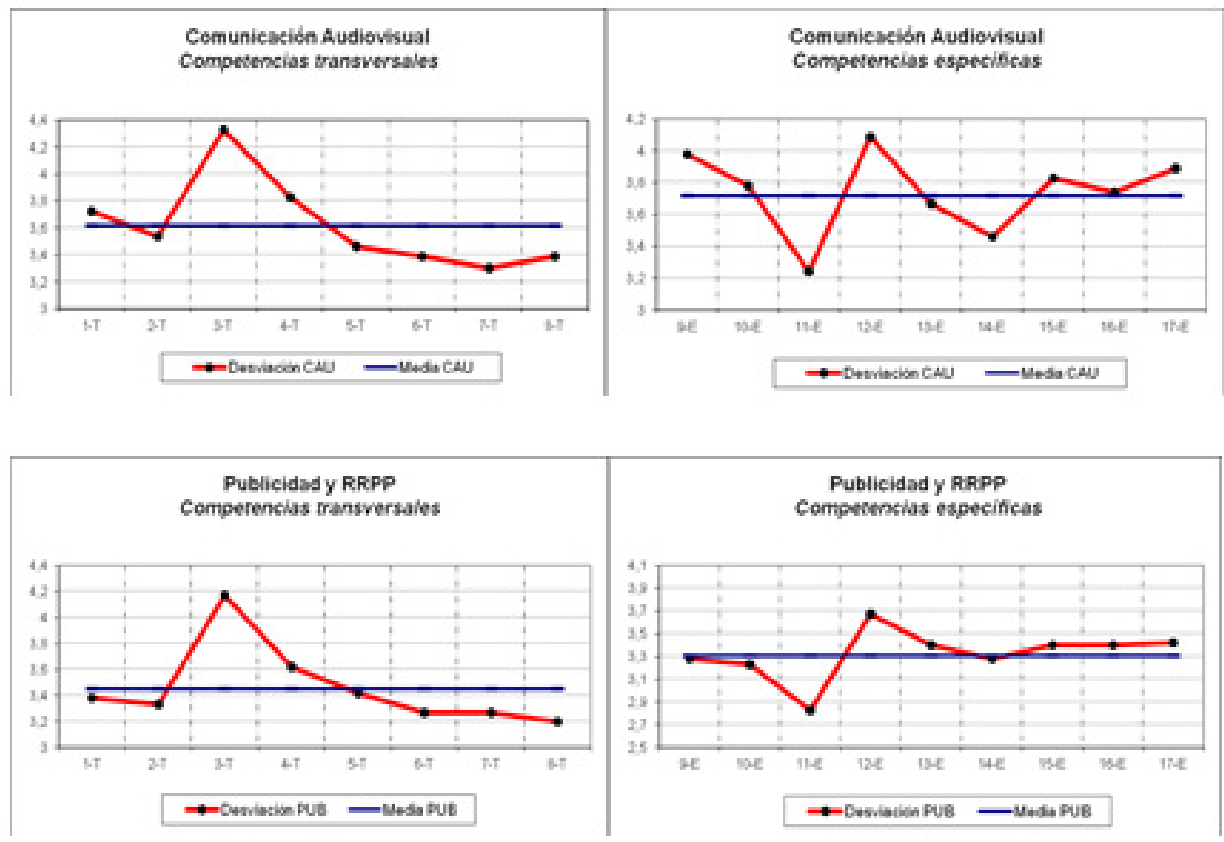

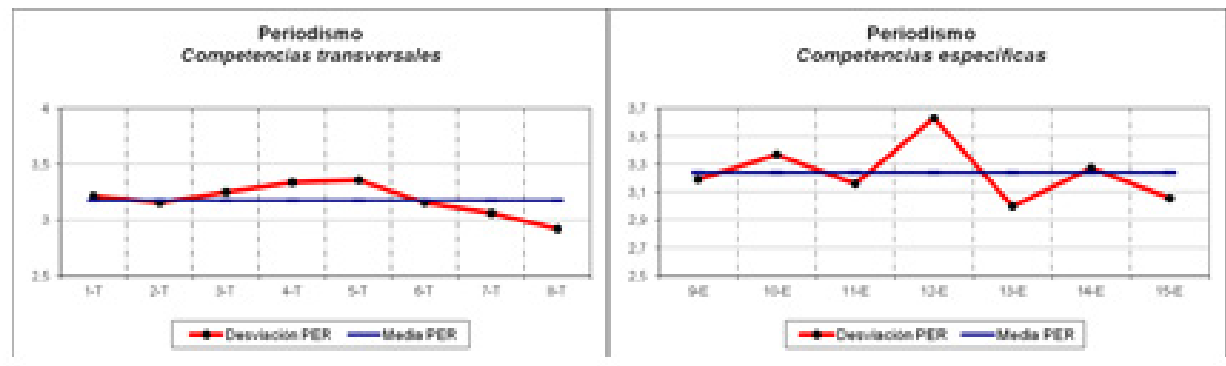

Por el contrario, los datos obtenidos para el Grado en Periodismo apenas varían en las competencias transversales, mientras que en cuanto a las específicas aumenta significativamente el diferencial de percepción para las competencias de carácter comprensivo y desciende para las relativas a la imaginación.

\subsection{Grupos de discusión}

Las opiniones de los estudiantes registradas en los grupos de discusión permiten observar tres aspectos de distorsión en el trabajo formativo mediante competencias. Así, por lo general, consideran excesivamente abstractas las competencias propuestas en los planes docentes, reconocen no contemplarlas en el devenir del periodo lectivo y ponen de manifiesto la dificultad para confirmar o refutar su adquisición.

\subsubsection{Competencias tranversales}

Por lo general, los estudiantes valoran su buen nivel de adquisición de competencias transversales, especialmente tras dos años de carrera, por lo que no las conciben como exclusivas de una u otra asignatura. En este sentido, coinciden en criticar la aparente redundancia de algunas competencias tranversales entre materias: "(la 2) Es más que nada para llenar la lista porque no ha habido ningún ejercicio que fuera en esa dirección" (Suj.3-PyRP). Y atribuyen a la falta de instrumentos de evaluación de determinadas competencias, el hecho de no ser propias ni originales de los estudios universitarios. Por ejemplo, destaca la idea de que en la universidad no se enseña a analizar y sintetizar, sino que se asume la habilidad sin evaluarla. Del mismo modo, el trabajo en equipo es una competencia previamente adquirida, sobre la que se evidencia cierta disparidad en las opiniones de los estudiantes en función de si el excesivo volumen de trabajo demandado los obliga a prescindir de esta dinámica. "La idea del trabajo en equipo es poder intercambiar opiniones. Pero como el tiempo disponible no da más de sí, acabas delegando tareas. Muchas veces el trabajo en equipo no es real" (Suj.3-CAU).

Esta disparidad de opiniones se traduce en una comprensión no unívoca de ciertas competencias. Así, por ejemplo, la capacidad de autocrítica (4), es reconocida 
como adquirida por todos los participantes de los diversos grupos de discusión, pero mientras que los estudiantes del Grado de Periodismo la adscriben a la reflexión promovida por el docente a través de la ejemplificación de casos informativos del mundo profesional o a su propia capacidad de observación ("Quiero decir, te fijas más, dices, mira éste, qué ha hecho ¿sabes? Al ver ejemplos y también al planteártelos ella..." Suj.7-PER), los otros dos grados remiten a la promoción de la crítica al trabajo desarrollado por los compañeros en el aula ("El hecho de que cuando un grupo está exponiendo un trabajo, otro grupo esté obligado a hacerle una pregunta, nos procura a nosotros mismos la capacidad de poder evaluar el trabajo de los demás y saber que lo harán con el nuestro" Suj.6-CAU). En cualquier caso, esta competencia, indiferenciable de la siguiente para los estudiantes, requiere, a su juicio, una retroalimentación evaluativa constante del docente.

Otro de los discursos críticos registrados entre los estudiantes es el que denuncia una atribución impropia del ámbito educativo universitario: los valores y la ética se enseñan en el entorno familiar o en fases previas de socialización, no en la universidad. Esto afecta, principalmente a las competencias vinculadas a la empatía y la inteligencia emocional (7) y la capacidad de actuar con libertad y corresponsabilidad (6). Si bien, para esta última, los estudiantes de Publicidad y Relaciones Públicas y los de Periodismo difieren en las causas de su nula o baja adquisición en el marco de la asignatura: el docente coarta su libertad como investigadores en las aplicaciones metodológicas de los primeros o es la dificultad de obtener una visión general de las perspectivas teóricas desde el inicio lo que les impide formarse su propia opinión a los segundos.

En esta misma línea, los estudiantes de Periodismo recriminan que el excesivo desarrollo teórico de la asignatura promueva la memorización de contenidos: Es tan abstracto que no te da tiempo a apuntar y además tener la capacidad de abstracción para aprender lo que te están explicando (Suj.7-PER). Mientras que por el contrario, los de Publicidad destacan la complejidad teórica de la materia como aliciente para aprender a aprender: Esto es porque no hemos tenido ningún contenido en la carrera que sea teórico y tienes ansias de que una cosa que no entendemos no sea yo quién no la entienda (Suj.4-PyRP).

\subsubsection{Competencias especificas}

Si bien los estudiantes reconocen la primera de las competencias específicas como centro de la asignatura, de nuevo, atribuyen a la falta de tiempo para la reflexión propia "del sistema industrial-educativo" (Suj.3-CAU), la dificultad para llegar a formarse una opinión crítica de las teorías.

A este discurso común se une, para los estudiantes de Comunicación Audiovisual y los de Publicidad, el de las ventajas en la asimilación de contenidos que comporta la aplicabilidad de la metodología, ya que la teoría se hace "más pesada" (Suj.3-CAU) cuando no "superficial" (Suj.8-CAU). Por el contrario, el contenido eminentemente teórico en Periodismo explica la mejor valoración del análisis crítico de fenómenos 
comunicativos y la búsqueda de claves interpretativas, competencias específicas que los otros dos grados opinan haber adquirido "en los seminarios y las clases prácticas, pero en las clases de teoría, no" (Suj.3-PyRP).

Así, el desarrollo de la imaginación y el pensamiento productivo es tan sólo apreciado por los estudiantes que complementan el contenido teórico con el metodológico: "Nos dijeron 'Haced un trabajo relacionado con los medios y que utilice una técnica vista anteriormente'. Entonces aqui puedes pensar, lo mejor que puedes estudiar o lo que es más interesante. Así que abres un poco la mente y te imaginas cosas" (Suj.7-PyRP). Mientras que la carencia de la carga metodológica en el desarrollo de la materia acentúa las dudas sobre la aplicabilidad de la teoría en los estudiantes de Periodismo ("Al final una asignatura se valora por si realmente te sirve a nivel práctico" Suj.3-CAU).

\section{Conclusiones y discusión}

El análisis de las competencias adquiridas por los estudiantes de una asignatura como Teorías de la comunicación es un proceso complejo, sobre todo si tenemos en cuenta el elevado número de competencias a evaluar y el hecho de que "las competencias se demuestran en la acción y, por lo tanto, sólo son evaluables si hay actividades de aplicación" (Gairín, 2009:17).

En nuestro caso, el método utilizado ha sido la medida indirecta a partir de la percepción que los propios estudiantes tienen de la adquisición de ellas, método que no está exento de limitaciones (Alonso et al. 2009:42-43). En primer lugar, la imprecisión y ambigüedad con que se define el concepto de competencia, que conlleva a la disparidad de acepciones registradas entre los estudiantes - habilidad, recurso, capacidad, conocimiento, actitud o herramienta, entre otras-. En segundo lugar, el incumplimiento de los dos requisitos básicos en la redacción de las competencias: comprensibilidad y transparencia (González y Wagenaar, 2003:53). Sobre este aspecto, si bien los estudiantes tienden a declarar que sí entienden las competencias sobre las que se les solicita su autoevaluación, se muestran contrariados por su carácter abstracto. Y es justamente esta falta de concreción percibida la que genera una actitud hacia las competencias de descrédito - "A mi me parece que los planes docentes son una formalidad muy banal" (Suj.3-PyRP)—, indiferencia y pragmatismo - "Yo al principio las iba mirando, no entendía absolutamente nada y pasé, voy directo a la evaluación y a lo práctico" (Suj.4-PER). En efecto, la ausencia de una comprensión unívoca y clara de las competencias a adquirir no sólo hace mucho más compleja la interpretación de los resultados obtenidos en este estudio, sino que introduce una ulterior crítica a los nuevos planes de estudio en el EEES: la inadecuación de los métodos de evaluación basados en la adquisición de competencias no suficientemente delimitadas o difícilmente evaluables en el curso lectivo de una materia. 
La detección de estos tres ejes problemáticos en la implementación de los planes docentes de Teorías de comunicación lleva, consecuentemente, a plantear tres líneas de actuación conjunta. La primera de ellas consiste en reducir el número de competencias a adquirir a través de la asignatura. Si la evaluación debe "reunir evidencia, en cantidad y calidad suficiente, para hacer juicios razonables acerca de la competencia de un individuo" (Villardón, 2006:63), es evidente que, por la limitación del tiempo que los estudiantes pueden dedicar a cada asignatura, el número de competencias debe ser reducido. La alternativa ha sido aumentar el trabajo en grupo, con el que se favorece la evaluación de una competencia en particular, pero se dificulta la evaluación del resto.

En segundo lugar, se plantea la posibilidad de unificar las herramientas de evaluación con el objetivo de concentrar en un menor número de pruebas el seguimiento del proceso de adquisición de competencias por parte del estudiante. Esta medida, acompañada del implemento de nuevas tecnologías -como las actividades de aprendizaje interactivo a través de Moodle- no sólo permitiría intensificar el contacto entre docente y discente y mejorar así la atención más personalizada que reclama el último, sino que también evidenciaría para ambos los factores clave de la adquisición de competencias y el proceso de evaluación como un continuo sobre el que intervenir.

En tercer lugar, se propone discriminar aquellas competencias que deben ser consideradas como no evaluables. Por un lado, se detectan algunas competencias que sólo podrán ser evaluadas posteriormente a la formación, cuando el estudiante empiece su actividad laboral (Gairín, 2009:28). Por otro lado, en la distinción establecida por los estudiantes en cuanto a las competencias exclusivas de la asignatura o compartidas entre diversas materias, parece evidente la necesidad de revisar cuáles son las competencias intrínsecamente propias de Teorías de la comunicación.

Aún así, esta revisión resulta más compleja en el ámbito de las competencias transversales, donde también los argumentos esgrimidos por los estudiantes se diversifican. Así, por una parte, es una opinión manifiesta y común entre los participantes en los grupos de discusión la impertinencia de ciertas competencias que deben darse por supuestas o ya adquiridas al acceder a los estudios universitarios. Mientras que, por otra parte, se registra una visión crítica respecto a ciertas competencias vinculadas a capacidades emocionales o éticas que no se aceptan como propias o legítimas de la educación universitaria. En este punto creemos que se debe afrontar plenamente el debate sobre la finalidad última de la universidad en el contexto actual -formación de profesionales aptos para el mercado laboral y/o ciudadanos capaces de participar responsablemente en el devenir social- que ha sido postergado por la urgente necesidad de implantar el EEES.

Por último, cabe destacar que las conclusiones de este estudio comparativo, más allá de las diferentes percepciones en la adquisición de las competencias que han sido detalladas para cada uno de los tres grados en comunicación de la Universidad Pompeu Fabra, permiten detectar cuál es el mayor reto para los docentes de Teorías de la comunicación. Y es que, si bien es apreciable la valoración positiva que los estu- 
diantes de Periodismo manifiestan respecto a la adquisición de competencias vinculadas a la capacidad de análisis crítico y reflexión sobre sus propias acciones, el hecho de que la asignatura en este grado no incluyera la parte metodológica, a diferencia de los otros dos grados, constituye una variable explicativa del menor grado de adquisición percibido en competencias específicas como la aplicación de conocimientos teórico-metodológicos.

En este sentido, los grupos de discusión han servido para comprobar cómo los estudiantes de los tres grados dudan de la aplicabilidad de la parte teórica de la asignatura. Habida cuenta que precisamente el EEES se diseñó como una estrategia de convergencia entre la universidad y el mercado de trabajo, y que los estudiantes perciben lo teórico como no aplicable, se ha pasado de considerar la competencia como el dominio de una disciplina a enfatizar la utilización práctica, cuantificable y medible (Barnett, 1999).

Tanto como readaptar los planes de estudio para hacerlos converger con las demandas de la empresas, es necesario que los estudiantes entiendan y asimilen el contenido teórico como algo útil y que les hace diferentes, como algo surgido, en la mayoría de las ocasiones, de la práctica, y que puede ser devuelto a este mismo terreno. La visión de los fenómenos comunicativos de un estudiante después de cursar la asignatura de Teorías de la comunicación no es la misma que la que tenía anteriormente. Y este saber es, sin duda, aplicable y altamente no obsolescente.

\section{Bibliografía}

ALONSO, L.E., FERNÁNDEZ, J.C. y NYSSEN, J.M. (2009). El debate sobre las competencias. Una investigación cualitativa en torno a la educación superior y el mercado de trabajo en España. Madrid: Aneca.

ÁLVAREZ, J.M. (2008). "Evaluar el aprendizaje en una enseñanza centrada en competencias" En GIMENO, J.(ed.), Educar por competencias ¿qué hay de nuevo? Madrid: Morata. p.206-233

ANGULO, J., CORPAS, C., GARCÍA, J.D., GONZÁLEZ, I., y MÉRIDA, R. (2006). "Las competencias de la titulación de psicopedagogía a nivel andaluz: investigando la opinión del profesorado, del alumnado universitario y de los profesionales de la orientación". En: Revista de Investigación Educativa, 24(2), p.575-594.

AQU Catalunya (2004). Marc general per a la integració europea. Barcelona: Agència per a la Qualitat del Sistema Universitari de Catalunya.

ARNOLD, J., LOGAN-CLARKE, J., HARRINGTON, A., y HART, C. (1999). "Students' perceptions of competence development in undergraduate business-related degrees". En: Studies in Higher Education, 24(1), p.43-59.

BAKX, A., VERMETTEN Y. \& VAN DER SANDEN, J. (2003). "Self-perceived competence, learning conceptions and preferred learning situations in the domain of communication". En: British Journal of Educational Psychology, 73, p.223245 . 
BARNETT, R. (1999). The limits of competence: Knowledge, higher education and society. Buckingham: Open University Press.

BIGGS, J. (2003). Teaching for quality learning at university. Buckingham: The Society for Research into Higher Education and Open University Press.

BONI, A. \& LOZANO, J.F. (2006). "The generic competences: an opportunity for ethical learning in the European convergence in higher education". En: High Educ, 54, p.819-83

DE LA CRUZ, M.A. (2003). "Necesidades y objetivos de la formación pedagógica del profesorado universitario". En: Revista de Educación, 331, p.35-66.

GAIRÍN, J. (ed.) (2009): Guía para la evaluación de competencias en el área de las ciencias sociales. Barcelona: AQU. http://www.aqu.cat/doc/doc_14646947_1.pdf [19-09-2013]

GIBBS, G. y SIMPSON, C. (2004). "Does your assessment support your students' learning?" En: Journal of Teaching and Learning in Higher Education, 1, p.3-32.

GONZÁLEZ, J. y WAGENAAR, R. (eds.) (2003): Tuning Educational Structures in Europe. Informe final. Universidad de Deusto. http://www.relint.deusto.es/ TUNINGProject/spanish/ [19-09-2013]

HILL, Y., LOMAS, L. y MACGREGOR, J. (2003). "Students' percepcions of quality in higher education". En: Quality Assurance in Educacion, 11(1), p.15-20.

KEMBER, D. y LEUNG, D. Y. P. (2005a). "The influence of active learning experiences on the development of graduate capabilities". En: Studies in Higher Education, 30, p.157-172.

KEMBER, D., y LEUNG, D. Y. P. (2005b). "The influence of the teaching and learning environment on the development of generic capabilities needed for a knowledge-based society" En: Learning Environments Research, 8, p.245-266.

LEUNG, D. Y. P. y KEMBER, D. (2005). "The influence of the part-time study experience on the development of generic capabilities". En: Journal of Further and Higher Education, 29, p.91-101.

LEUNG, D. Y. P. y KEMBER, D. (2006). "The influence of teaching approach and teacher-student interaction on the development of graduate capabilities". En: Structural Equation Modelling, 13, p.164-186.

MARTÍNEZ-COCÓ, B., GARCÍA, J.N., ROBLEDO, P., DÍEZ, C., ÁlVAREZ, M.L., MARBÁN, J.M., DE CASO, A.M., FIDALGO, R., ARIAS-GUNDÍN, O., PACHECO, D.I., y RODRÍGUEZ, C. (2008). "Valoración Docente de las Metodologías Activas: un Aspecto Clave en el Proceso de Convergencia Europea". En: Aula Abierta, 35 (1-2), p.35-48.

MINISTERIO DE EDUCACIÓN CULTURA Y DEPORTE (2003). La integración del sistema universitario español en el espacio europeo de enseñanza superior. Documento Marco. Madrid: Ministerio de Educación, Cultura y Deporte.

PARAHOO, K. (1999). Nursing research: principles, problems and issues. London: Macmillan.

PEDRÓ, F.(dir.) (2005). Manual d'introducció a la docència. Horitzó Bolonya, Barcelona: UPF 
RAUHVARGERS, A. (2011). "Achieving Bologna Goals: Where Does Europe Stand Ahead of 2010". En: Journal of Studies in International Education, 2011, 15:4

STIGGINS, R. (2006). "Assessment for learning: A key to motivation and achievement". En: Edge, 2(2), p.3-19.

UPF (2009). L'organització de la docència a la UPF. Barcelona: UPF

VILLARDÓN, L. Evaluación del aprendizaje para promover el desarrollo de competencias. Educatio Siglo XXI, 2006, no 24, p. 57-76. http://revistas.um.es/index. php/educatio/article/viewFile/ 153/136 [19-09-2013]

ZABALA, A. y ARNAU, L. (2008). Once ideas clave: cómo aprender y enseñar competencias. Barcelona: Graó.

\section{Los autores}

Lorena Gómez-Puertas es licenciada en Comunicación Audiovisual y Doctora en Comunicación por la Universidad Pompeu Fabra. Es profesora del Departamento de Comunicación de la misma universidad, donde imparte las materias de Teorías de la comunicación y de la cultura, Opinión pública, Comunicación política y Análisis del contenido y discurso. Sus líneas de investigación se centran en la construcción mediática de los discursos sociales, en particular a través de la ficción televisiva; la comunicación política; y la educación en el ámbito de las teorías de la comunicación y la cultura. Ha publicado artículos en todas estas áreas.

Carles Roca-Cuberes es licenciado en psicología por la Universitat Autònoma de Barcelona y doctor en sociología por la Universidad de Manchester (Gran Bretaña). Es profesor del Departamento de Comunicación de la Universidad Pompeu Fabra, donde enseña teorías de la comunicación y metodología de la investigación social. Ha publicado artículos en la campo de la comunicación, tanto interpersonal como mediada, en revistas internacionales; también ha publicado y llevado a cabo diversos estudios en el ámbito de la educación universitaria.

Frederic Guerrero-Solé es doctor en Comunicación Social por la Universidad Pompeu Fabra. Actualmente trabaja como profesor ayudante del Departamento de Comunicación de la misma universidad, donde imparte las materias de Sociología de la comunicación, Comunicación intercultural, Delincuencia y medios de comunicación, y Métodos cuantitativos de investigación. Sus líneas de investigación comprenden la sociología de la comunicación, los efectos de los medios y los grandes acontecimientos mediáticos, además de los medios en Rusia. Ha publicado artículos en todas estas áreas. 